\title{
Erratum to: For Black Models Scroll Down: Webcam Modeling and the Racialization of Erotic Labor
}

\author{
Angela Jones ${ }^{1}$
}

Published online: 26 October 2015

(C) Springer Science+Business Media New York 2015

\section{Erratum to: Sexuality \& Culture (2015) 19:776-799 \\ DOI 10.1007/s12119-015-9291-4}

Unfortunately, the Web site was not updated in the original publication, and it has been corrected with this erratum.

1. On page 776 , the fifth sentence in the Abstract should read as:

"First, the article presents descriptive data based on participation observation on the Web site of focus myfreecams.com"

2. On page 779 , the footnote 1 and its citation should be deleted. The corrected sentence should read as:

"December 22, 2013, was a typical night on myfreecams.com logged on at 8:00 pm-a popular time on the Web site"

3. The third line of the second paragraph in the Introduction should read as:

"First, the article presents descriptive data based on participation observation on the Web site of focus myfreecams.com."

The online version of the original article can be found under doi:10.1007/s12119-015-9291-4.

Angela Jones

jonesa@farmingdale.edu

1 Farmingdale State College, State University of New York, 2350 Broadhollow Rd, Memorial Hall, Rm 124, Farmingdale, NY 11735, USA 